\title{
Quality of Nursing Care in Saudi Arabia: Needs for Improvement
}

\section{Abbas Al Mutair}

Mohammed Al Mana College for Health Sciences, Ministry of Education, Saudi Arabia

*Corresponding author: Dr. Abbas Al Mutair, Assistant Professor at Mohammed Al Mana College for Health Sciences, Ministry of Education, Saudi Arabia, Tel: 00966555877487; E-mail: abbas4080@hotmail.com

Rec date: Sep 14, 2015; Acc date: Oct 21, 2015; Pub date: Oct 29, 2015

Copyright: $\odot 2015$ Abbas Al Mutair. This is an open-access article distributed under the terms of the Creative Commons Attribution License, which permits unrestricted use, distribution, and reproduction in any medium, provided the original author and source are credited.

\section{Abstract}

Aims: To discuss the need to improve the quality of health care by nurses as well as the need to improve safety in the delivery of patient care and evidence based nursing practice in Saudi Arabia.

Background: Quality improvement in Saudi Arabia has become a major focus within health care, especially in the areas of regulatory quality, quality assurance, quality improvement and patient safety. The issues and trends affecting nursing care today are increasingly complex and dynamic.

Results: The need to improve quality is highly required in Saudi Arabian health care settings because many shortcomings are being reported every day like nursing and medical errors. The lack of quality is due to many reasons such as the absence of positive organizational climate and culture, no clear mission, vision, values and policies. The abovementioned weaknesses in quality improvement and patient safety emphasize the significance of evidence based practice in order to study their origins and overcome them. The implementation of evidence based practice would ultimately helps in promoting the professional image of nursing in the hospitals in Saudi Arabia and accordingly improves the nursing and hospital performance.

Conclusion and implications: The nursing departments should obligate to provide all people of Saudi Arabia a safe and caring environment and optimum nursing services to patients. The nursing departments are also required to demonstrate the highest quality of care supported by professional development. This should be collaborated with the other healthcare providers and supported by evidence based practice. Nurses provide the majority of patient care, they must be empowered to continuously improve care and service for meeting quality of patient care.

\section{Key words:}

Evidence-based Practice; Nursing, Patient Safety; Saudi Arabia; Quality Improvement

\section{Quality improvement}

There is no doubt of the great need for nurses to improve the quality of health care delivery. One of the reasons that improving the quality of health care delivery is that it can increase the cost effectiveness of nurses in various health care settings. Errors are a problem for hospitals and other health care organizations because they cost life and money [1]. For example, in practice there is very little supervision of the non-licensed or new staff nurses by professional nurses in most of the Ministry of Health hospitals in Saudi Arabia, therefore many medical and nursing errors are being reported. These cause harms to patients, cost money and take extra staff time since every time an error is made by a nurse, it must be reported. The report writing alone increases the load and takes the nurse away from the patient [1].

In view of the above, it is important to understand two elements that contribute fundamentally in shaping the organizational framework of performance in a positive or negative manner. These two elements are the organizational climate and the culture; accordingly it is significant to distinguish between them. According to Ellis and Hartely organizational climate "refers to the perception of an organization with regard to the prevailing feelings and values of the organization" (2005, p. 63). Also, feelings of trust, belonging, esteem and loyalty would be some of the organizational climate examples [2]. Moreover, when a positive climate exists in a work setting with relation to its goals and staff needs, it may create high quality performance and increased motivation among workers towards improving the final outcomes [3]. When the nurse manager for example engages the employees in the decision making process, it is likely to improve their performance as they find themselves as partners, not just staff.

The organizational climate is influenced by the organizational culture [2]. The culture is the customary way of thinking and behaving that is shared by all members of the organization and must be learned and adopted by newcomers [4]. Also, culture is learned, shared and transmitted; it is a combination of values, language, behavior and beliefs [4]. Therefore, understanding the organizational culture is vital to improve the organization's performance and helps to effectively attain its goals. For instance, if the nurses who come from overseas understand the Saudi culture and speak Arabic language that would help them to easily adapt to the changes they encounter and accomplish the organization's goals.

Thus, there is no doubt of the significance of the policies, mission, vision and values in any health care organizations. According to Gracher and Coffexy in 1993 one of the fundamentals of quality improvement, suggests that organization must have an overriding competitive vision. A clear mission, vision, values and policies would act as a pathway for the health workers. For example, the clear job 


\section{(20.4172/2167-1168.1000309}

Page 2 of 3

description for any position would direct the nurses toward the main mission and restrict their job within the profession. However, many the Ministry of Health hospitals do not have clear vision to be achieved which can improve the nursing function and quality as well. Moreover, it is popular to find hospitals that have been working for years and do not have policy and procedure manual, or they may have one but it is outdated and needs to be reviewed. Hence, it is vital for the hospitals to have sustainable long term mission, vision, values to be achieved and policies to improve the quality of nursing care.

Nurses in Saudi Arabia also encounter a number of challenges and difficulties which may prevent them from achieving their tasks with regards to patient care in clinical areas. For example, the rapid increase of patients' admission without a rational increase in posting new staff causes severe shortages in nurses in many hospitals. Moreover, most of the secretary work such as typing and letters follow up is delegated to the nurses. Subsequently, this may redirect the nurses' efforts from the main mission to other non-professional tasks, which gives less time for patient care and focusing on the main goals. To avoid taking nurses away from their main jobs, all secretarial work should be done by assigned secretaries

Professional development of the nurses would ultimately lead to quality improvement and subsequently the improvement of the services offered by the hospital. Many of the world's countries have restricted the nursing education to not less than bachelor degree. However, in Saudi Arabia the hospital nursing staff composes mostly of diploma holder which may possibly have negative effect on the care being delivered to the patients. According to Aiken, Clarke, Cheung, Sloane and Silber in 2003 in hospitals with higher proportions of nurses educated at bachelor level or higher, patients may experience lower failure to patient care. Moreover, professional development such as attending conferences, seminars and workshops would strengthen the nurse's self confidence and increases their independency and improves their performance. The need for the high qualification and professional development of nurses in Saudi Arabia cannot be overemphasized. High qualification and professional development of the nurses would ultimately lead to improvement of the services offered by the hospital and specifically to the quality of patient care provided by the nurses.

\section{Safety and performance improvement}

The need to improve patient safety for continuous quality improvement has been extensively recognized as a key priority in many countries over the entire world. Byers and White in 2004 argue that adverse events are a leading cause of death. Patient safety must be every health professional's responsibility because malpractice in the health care settings can result in serious harm [5]. As nurses are the last line of defense between the healthcare system and the patient [5] for they need to be aware and have the abilities and knowledge to ensure patient safety. However, this is not the case in a number of the hospitals in Saudi Arabia. Many medical errors are being reported in different hospitals in the kingdom without monitoring and corrective action by the administration channels. This negatively impacts on the patient safety and the quality improvement hindered and the ultimate patient care delivery goal is ignored. For example, this is a case which happened in the operating theatre at X hospital in Saudi Arabia. The evening charge nurse is the one responsible for checking supplies and restocking them according to department policy. One day, the charge nurse checked the ophthalmology theatre's disinfection iodine stock and found that it was empty and it needed to be refilled. The iodine which is used for eye disinfection is $1 \%$ but the nurse refilled the $1 \%$ iodine containers with $10 \%$ iodine which is usually used for hands washing. As a result, the nurses were using the $10 \%$ iodine instead of the $1 \%$ iodine for disinfecting the patient's eyes for the whole next week. Although, this was caused by staff nurses, the nursing leadership did not take action to improve the process to prevent such mistakes which violate patient safety. There was no follow up action either.

In another instance, the absence of administration awareness of another major trauma hospital resulted in Acinetobactor outbreak which started with 14 patients in the Intensive Care Unit. Then the bacteria spread to the other hospital admission departments reaching 24 infected cases. Consequently, the hospital lost its reputation and people's confidence. However, the epidemic's origin is due to many reasons such as the rapid increase of daily admission especially since this is the only major trauma hospital and serves 1,250,000 of population. Further reasons staff shortage and staff malpractice as well as reasons related to lack of hospital stock supplies by the hospital's administration like hand sanitizer, a disinfectants and tissues. Nevertheless, all of the causes appear to be avoidable if the organization's administration took an active part in patient's safety and gave better consideration in protecting patients from any danger that might occur before it happened.

Additionally, there is increasing evidence in the literature that patient outcomes are strongly linked to nursing performance and the independent decisions nurses make regarding the assessment, treatment and nursing interventions performed on patients have significant impact on quality of care [6]. Nevertheless, standardization of medical and nursing care plans in these hospitals is not in effect and critical paths are not used. Therefore, a number of medical and nursing errors are being reported. Also, nursing leadership and management roles are not prominent, and in view of that there are some managerial troubles and interruptions between the different levels of nursing staff. Consequently, there are some issues which have lowered the nursing performance and as a consequence hospitals' performance. This draws attention to the need for patient safety and performance improvement in the delivery of health care by nurses in Saudi Arabian health organizations.

\section{Evidence-based nursing practice}

Education and research should be valued by nurses in Saudi Arabia and their participation must demonstrate pride and commitment to the nursing profession. Nurses are responsible and accountable for their practice and they should provide evidence of standards of the care they provide [1]. Nurses are required to develop their knowledge and skills in line with the latest available evidence. On the other hand, nurses often make decisions about patient care based on their personal experience or tradition rather than scientific evidence. For example, hospital policies are often written by the experienced nursing staff so it is usually based on their experience rather than scientific evidence. However, trial and error is another source of evidence for many nurses according to Schmidt and Brown in 2009. Although, this approach is not effective as it can reduce nurses' critical thinking and wastes time and resources [7]. They also argue that this is because trial and error is not based on a systematic scientific approach, which may lead to malpractice [7].

Furthermore, to appreciate the importance of evidence for nurses a survey initiated by the staff developmental coordinator was carried out on the nurses of a major hospital. The survey intended to examine the 
Citation: Mutair AA (2015) Quality of Nursing Care in Saudi Arabia: Needs for Improvement. J Nurs Care 4: 309. doi:

Page 3 of 3

nurses' favorite site for giving an intramuscular injection. The vast majority of the nurses responded that they give the injection in the buttocks and many continue to use the four-quadrant method to locate the injection site. They rarely mentioned the ventrogluteal site for giving an IM injection, which is the safest as it is away from the nerves and most accessible site to use according to Perry in 2001. In addition, when nurses were asked how they positioned the patient, most responded that they asked the patient to lie on his or her side, since the late seventies and early eighties [8]. Though, research has supported the prone position as the most comfortable position for the patient giving an IM in this site [8]. Such practices are still being taught by the nursing schools in the Kingdom.

Evidence can be used routinely for decision making in healthcare settings. Based on the aforementioned issues the Ministry of Health which is the principal governing body of most hospitals in Saudi Arabia may use evidence in many practices. Practices may include for example, understanding and observation of these shortcomings of past practices, improving nursing quality, planning for infection control. The Study of these problems would provide the hospitals authorities solutions for these frequent troubles. Moreover, the Ministry of Health is required to encourage and support evidence based practice. Still this is not the case and it is not possible to get funding for conducting research unlike the developed countries such as United States of America, Canada and Australia.

Furthermore, because books are often outdated by the time they are published, many believe that nurses can no longer rely on textbooks only to provide the latest and the best information to guide their practice [9]. On the other hand, the hospitals authorities are not committed to provide the nurses with the latest available journals. This may resulted in lack of capacity to demonstrate the highest quality of care supported by professional development. By and large, the advanced development of nursing care could be hindered due to the aforementioned reasons which seem not to appreciate the significance of evidence based practice.

\section{Relevance to nursing practice}

The nursing profession in Saudi Arabia should commit to provide a safe and caring environment and optimum nursing services to patients. Nurses are also required to demonstrate the highest quality of care supported by professional development. This should be collaborated with the other healthcare providers and supported by evidence based practice.

\section{Conclusion}

The need to improve quality is highly required in Saudi Arabian health care settings because many shortcomings are being reported every day like nursing and medical errors. The lack of nursing quality is due to many reasons such as the absence of positive organizational climate and culture, no clear mission, vision, values and policies. Also, the lack of nursing professional development and redirecting the nurses to non professional tasks has acted as an obstacle to improve quality. Additionally, due to the frequent violating of patients' safety and performance in health care settings, these improvements should be obligatory. The abovementioned weaknesses in quality improvement and patient safety emphasize the significance of evidence based practice in order to study their origins and overcome them. The implementing of evidence based practice would ultimately helps in promoting the professional image of nursing in the hospitals in Saudi Arabia and accordingly improves the nursing and hospital performance

\section{References}

1. Morrel C and Harvey G (1999) The clinical audit handbook: improving the quality of health care. Bailliere Tindall, London.

2. Ellis J. and Hartley C. (2005). Managing and coordinating nursing care (4th ed.). Lippincott Williams \& Wilkins, Philadelphia.

3. Ellen M. Gohar-Murray and DiCroce H. (2003). Leadership and management in nursing (3rd ed.). Person Education, New Jersey.

4. Tomey A (2000). Guide to nursing management and leadership (6th ed.). Mosby, St Louise.

5. Attree M, Cooke H, Wakefield A (2008) Patient safety in an English preregistration nursing curriculum. Nurse Educ Pract 8: 239-248.

6. Rice S.M, Slobbe A.V, and Rathgeber D. (2007). Nursing practice evaluation using an expert panel process. Clinical Governance 12: 93-101.

7. Schmidt N. A, and Brown J. M. (2009). Evidence-based practice for nurses: appraisal and application of research. Jones and Bartlett Publisher, Ontario.

8. Perry P. (2001) Fundamentals of nursing (5th ed.). Mosby, St. Louis.

9. Levin R. f, and Feldman H. R. (Eds.). (2006). Teaching evidence-based practice in nursing. Springer, New York. 